\title{
ON NONCONTINUOUS CHAOTIC FUNCTIONS
}

\author{
JACK CEDER
}

(Communicated by Andrew M. Bruckner)

\begin{abstract}
A function $f:[0,1] \rightarrow[0,1]$ is constructed such that for each two distinct points $x$ and $y$ in $[0,1]$ the sequence $\left\{\left|f^{n}(x)-f^{n}(y)\right|\right\}_{n=0}^{\infty}$ is dense in $[0,1]$. Here $f^{n}$ is the $n$th iterate of $f$. Moreover a Baire 2 function can be constructed so that the above condition is valid for all distinct $x$ and $y$ in a dense open subset of $[0,1]$.
\end{abstract}

Let $f$ be a function from $I=[0,1]$ into $I$. The iterates of $f$ are defined inductively by $f^{0}(x)=x$ and $f^{n+1}(x)=f\left(f^{n}(x)\right)$. Following the definition of [2] we say a subset $S$ of $I$ is scrambled for $f$ (or $f$ scrambles $S$ ) provided

$$
\limsup _{n \rightarrow \infty}\left|f^{n}(x)-f^{n}(y)\right|=1
$$

and

$$
\liminf _{n \rightarrow \infty}\left|f^{n}(x)-f^{n}(y)\right|=0
$$

for any distinct points $x$ and $y$ of $S$. The function $f$ is called chaotic when $S$ is uncountable. Hence, for chaotic functions, orbits of points that are close together can be arbitrarily close together or far apart depending on the place in the orbit that is measured.

Many articles have appeared in recent years showing rather simple continuous functions that can scramble rather "big" sets (see [4, 2] and their bibliographies). In fact, [2] has improved earlier results and has shown that a continuous function cannot scramble an open set or a residual set, but it can scramble an $F_{\sigma}$ set of full measure or a set that is second category in each interval.

It is also shown in [2] that under the assumption of the continuum hypothesis a continuous function $f$ can scramble a set $T$ that is everywhere of second category with the additional property that the sequence $\left\{\left|f^{n}(x)-f^{n}(y)\right|\right\}_{n=0}^{\infty}$ is dense in $I$ whenever $x$ and $y$ are distinct members of $T$. If $f$ has this property we will say that $f$ wildly scrambles $T$.

Received by the editors January 31, 1990 and, in revised form, April 9, 1990.

1980 Mathematics Subject Classification (1985 Revision). Primary 54H20, 26A18. 
The purpose of this article is to initiate a study of scrambled sets relative to noncontinuous functions. ${ }^{1}$ One would suspect that by relaxing the requirement of continuity, bigger scrambled sets could appear.

In particular, can a function scramble all of $I$ ? If so, can it be a Baire $\alpha$ function? If not, can a Baire $\alpha$ function scramble an open set or a residual set?

Our main result, Theorem 2 , shows that a function can wildly scramble all of $I$. Whether such a function can be a Baire $\alpha$ function or Lebesgue measurable remains an open question. We also show that there exists a Baire 2 function that scrambles a dense open set. It is an open question whether or not such a function can be a Baire 1 function. We begin with the latter result.

Theorem 1. There is a Baire 2 function $h$ from $I$ onto $I$ that scrambles a dense open set.

Proof. According to Theorem 3.1 of [2] the "hat" function $f$ scrambles an uncountable $G_{\delta \sigma}$ set. In particular $f$ scrambles some nowhere dense perfect set $P$ where $a=\inf P>0$ and $b=\sup P<1$. Let $Q$ consist of $P$ minus $a, b$ and minus the left-hand endpoints of the components of $[a, b]-P$. Let $\left\{a_{n}\right\}_{n=1}^{\infty}$ and $\left\{b_{n}\right\}_{n=1}^{\infty}$ be sequences in $(0, a)$ and $(b, 1)$ respectively such that $a_{n} \rightarrow 0$ and $b_{n} \rightarrow 1$. Let $F_{1}$ be the countably infinite set containing $0,1, a, b$, each $a_{n}$, each $b_{n}$, and each endpoint of a component of $[a, b]-P$. Let $\mathscr{G}$ be the set of components of $I-F_{1}-Q$. Enumerate $\mathscr{G}$ as $\left\{G_{n}\right\}_{n=1}^{\infty}$ such that $G_{3 k} \subseteq(0, a), G_{3 k+1} \subseteq(b, 1)$, and $G_{3 k+2} \subseteq(a, b)$ for all $k$. Then $G_{3 k} \rightarrow 0$ and $G_{3 k+1} \rightarrow 1$.

Now choose a Cantor set $T$ in $[a, b]$ such that $T \cap P=\{a, b\}$. Let $\mathscr{H}$ be the set of components of $[a, b]-T$. For each $H \in \mathscr{H}$ for which $H \cap Q \neq \varnothing, H \cap Q$ is a dense, boundary $G_{\delta}$ subset of $P$ and hence by [3, p. 442] homeomorphic to $N$, the set of irrationals in $I$. As $Q$ is a dense $G_{\delta}$ subset of $P, Q$ is homeomorphic to $N$. Hence, we may decompose $Q$ into mutually disjoint sets $\left\{Q_{n}\right\}_{n=1}^{\infty}$ such that each $Q_{n}$ is homeomorphic to $Q$.

Now let $C$ be the Cantor ternary set in $I$ and $\left\{J_{n}\right\}_{n=0}^{\infty}$ the sequence of its complementary intervals. Let $F_{2}$ consist of 0,1 and all the endpoints of the $J_{n}$-intervals. Then, as above, we may decompose $C-F_{2}$ into mutually disjoint sets $\left\{S_{n}\right\}_{n=0}^{\infty}$ such that each $S_{n}$ is homeomorphic to $N$ and hence to $Q$. Moreover, we can do this in such a way that $S_{3 k} \rightarrow 0$ and $S_{3 k+1} \rightarrow 1$.

We can map $Q$ onto $(0,1)$ by the Cantor function $\gamma$ such that $\gamma^{-1}$ is Baire 1 (see [3, p. 442]). It follows that there is a continuous function $h_{n}$ on $I$ mapping $Q_{n}$ onto $J_{n}$ such that $h_{n}^{-1}$ is Baire 1. Likewise we can find a continuous function $k_{n}$ on $I$ mapping $S_{n}$ onto $G_{n}$ such that $k_{n}^{-1}$ is Baire 1 . Pick $s$ to be any 1-1 function on $I$ mapping $F_{1}$ onto $F_{2}$ such that $s(0)=0$, $s(1)=1$ and $s$ is continuous at 0 and 1 .

Identifying a function with its graph we put

$$
g=\left(\bigcup_{n=0}^{\infty} h_{n}\right) \cup\left(\bigcup_{n=0}^{\infty} k_{n}^{-1}\right) \cup s .
$$

\footnotetext{
${ }^{1}$ A study of $\omega$-limit sets (that is, sets of subsequential limits of the orbits $\left\{f^{n}(x)\right\}_{n=0}^{\infty}$ where $x \in I$ ) for noncontinuous functions was conducted in [1].
} 
Then $g$ is a $1-1$ function from $I$ onto $I$. If $G$ is open,

$$
g^{-1}(G)=\left(\bigcup_{n=0}^{\infty}\left[h_{n}^{-1}(G) \cap Q_{n}\right]\right) \cup\left(\bigcup_{n=0}^{\infty} k_{n}(G) \cap G_{n}\right) \cup\left(s^{-1}(G) \cap F_{1}\right)
$$

which is clearly an $F_{\sigma}$ set. Likewise

$$
\begin{aligned}
\left(g^{-1}\right)^{-1}(G) & =g(G) \\
& =\left(\bigcup_{n=0}^{\infty}\left(h_{n}(G) \cap J_{n}\right)\right) \cup\left(\bigcup_{n=0}^{\infty} k_{n}^{-1}(G) \cap S_{n}\right) \cup\left(s(G) \cap F_{2}\right)
\end{aligned}
$$

is an $F_{\sigma}$ set. Therefore both $g$ and $g^{-1}$ are Baire 1 functions. Moreover, it is clear that $g$ is continuous at both 0 and 1 .

Now define $h=g \circ f \circ g^{-1}$. Then $h$ is a Baire 2 function from $I$ into $I$ (see [3, p. 376]). Moreover, $g^{-1} \circ h^{n}=f^{n} \circ g^{-1}$ for all $n$.

For $x$ and $y$ distinct in $I-C, g^{-1}(x)$ and $g^{-1}(y)$ belong to $Q$ since $g(Q)=I-C$. Hence, the sequence $\left\{\left|g^{-1} \circ h^{n}(x)-g^{-1} \circ h^{n}(y)\right|\right\}_{n=0}^{\infty}=$ $\left\{\left|f^{n} \circ g^{-1}(x)-f^{n} \circ g^{-1}(y)\right|\right\}_{n=0}^{\infty}$ has cluster values 0 and 1 . Since $g$ is continuous at 0 and 1 , it follows that $\left\{\left|h^{n}(x)-h^{n}(y)\right|\right\}_{n=0}^{\infty}$ has cluster values of 0 and 1 . For example if 1 is a cluster value of $\left\{\left|g^{-1} \circ h^{n}(x)-g^{-1} \circ h^{n}(y)\right|\right\}_{n=0}^{\infty}$ then there is a subsequence $\left\{k_{n}\right\}_{n=0}^{\infty}$ for which $g^{-1} h^{k_{n}}(x) \rightarrow 0$ and $g^{-1} h^{k_{n}}(y) \rightarrow 1$ (or vice versa with $x$ and $y$ ). Then $h^{k_{n}}(x) \rightarrow g(0)=0$ and $h^{k_{n}}(y) \rightarrow g(1)=1$. Likewise 0 is a cluster value. This completes the proof.

The first step in proving Theorem 2 is constructing a function that wildly scrambles the set of rationals.

Lemma 1. Let $J$ be the set of rationals in $I$. Then there exists a $g$ from $J$ into $J$ such that for any distinct $x$ and $y$ in $J,\left\{\left|g^{n}(x)-g^{n}(y)\right|\right\}_{n=1}^{\infty}$ is dense in $I$.

Proof. Let $\left\{r_{i}\right\}_{i=0}^{\infty}$ be an enumeration of $J$. For. $x \in J, r^{-1}(x)$ is that $i$ such that $x=r_{i}$. Put $E=\left\{\left(r_{i}, r_{j}, r_{n}, k\right): i \neq j, k \in \omega_{0}-\{0\}\right\}$. Enumerate $E$ as $\left\{e_{m}\right\}_{m=0}^{\infty}$ and assume for convenience that $e_{0}=\left(r_{0}, r_{1}, r_{1}, 1\right)$. Put $e_{m}=$ $(\alpha(m), \beta(m), \gamma(m), \xi(m))$.

We will define by induction sets $C_{m}$ and $g_{m}$ for each $m \in \omega_{0}$ satisfying the following properties:

(1) $C_{m}$ is a finite subset of $J$ containing $\alpha(m)$ and $\beta(m)$;

(2) $g_{m}$ is a 1-1 function from $C_{m}$ into $J$;

(3) $g_{m} \subseteq g_{m+1}$;

(4) if $x \in C_{m}$, then $x \in J-g_{m}\left(C_{m}\right)$ or there exist $i$ and $j$ such that $g_{m}^{i}\left(r_{j}\right)=x$ and $g_{m}^{s}\left(r_{j}\right) \in C_{m}$ for all $s<i$

(5) there exists $v$ such that $\mid\left(g_{m}^{v}(\alpha(m))-g_{m}^{v}(\beta(m))-\gamma(m) \mid<(\xi(m))^{-1}\right.$; and

(6) $r^{-1}\left(g_{m}(x)\right)>r^{-1}(x)$ for all $x \in C_{m}$. 
For $m=0$ we have $e_{0}=\left(r_{0}, r_{1}, r_{1}, 1\right)$. Put $C_{0}=\left\{r_{0}, r_{1}\right\}$ and select $a \in J-C_{0}$ such that $a<1$. Then put $g_{0}\left(r_{0}\right)=r_{1}$ and $g_{0}\left(r_{1}\right)=a$. Conditions (1) through (6) are then satisfied.

Now assume we have defined $C_{m}$ and $g_{m}$ satisfying the inductive hypothesis.

Let $e_{m+1}=\left(r_{i}, r_{j}, r_{n}, k\right)$. By (4) it follows that $r_{i} \in J-g_{m}\left(C_{m}\right)$ or there exists a greatest $s \geq 0$ such that $g_{m}^{s}\left(r_{i}\right) \in g_{m}\left(C_{m}\right)$. Likewise $r_{j} \in J-g_{m}\left(C_{m}\right)$ or there exists a greatest $t \geq 0$ such that $g_{m}^{t}\left(r_{j}\right) \in g_{m}\left(C_{m}\right)$.

Consider the case when both $s$ and $t$ exist. Without loss of generality we may assume that $s=t+u$ with $u \geq 0$. Pick distinct points $b_{1}, b_{2}, a_{1}, a_{2}, \ldots, a_{u+2}$ in $J-C_{m}-g_{m}\left(C_{m}\right)$ so that

$$
\begin{aligned}
& i<r^{-1}\left(b_{1}\right)<r^{-1}\left(b_{2}\right) \text { and } j<r^{-1}\left(a_{1}\right)<r^{-1}\left(a_{2}\right)<\cdots<r^{-1}\left(a_{u+2}\right) \text { and } \\
& \left|\left(b_{2}-a_{u+2}\right)-r_{n}\right|<1 / k \text {. }
\end{aligned}
$$

Define $C_{m+1}=C_{m} \cup g_{m}\left(C_{m}\right) \cup\left\{b_{1}, a_{1}, a_{2}, \ldots, a_{u+1}\right\}$ and $g_{m+1}=g_{m} \cup$ $\left\{\left(g_{m}^{s}\left(r_{i}\right), b_{1}\right),\left(g_{m}^{t}\left(r_{j}\right), a_{1}\right),\left(b_{1}, b_{2}\right)\left(a_{1}, a_{2}\right), \ldots,\left(a_{u+1}, a_{u+2}\right)\right\}$.

In case $s$ or $t$ does not exist then $r_{i}$ or $r_{j}$ misses $g_{m}\left(C_{m}\right)$ and we can replace in the above construction $g_{m}^{s}\left(r_{i}\right)$ or $g_{m}^{s}\left(r_{j}\right)$ by $r_{i}$ or $r_{j}$ respectively and consider $s$ or $t$ to be 0 .

Clearly conditions (1) through (6) are satisfied, completing the induction. Put $g=\bigcup_{m=0}^{\infty} g_{m}$. Then it is easily verified that $g$ is a $1-1$ function from $J$ onto $J-\left\{r_{0}\right\}$.

Let $x, y, z$ by any points in $J$ with $x \neq y$ and let $n \in \omega_{0}$. Then $(x, y, z, n+1)=e_{m}$ for some $m$ so by (5) there exists $v$ such that $\mid\left(g^{v}(x)-\right.$ $\left.g^{v}(y)-z\right) \mid<(n+1)^{-1}$. It follows that $\left\{\left|g^{k}(x)-g^{k}(y)\right|\right\}_{k=1}^{\infty}$ is dense in $I$. Moreover, observe that from (6) we have $i<r^{-1}\left(g\left(r_{i}\right)\right)$ for each $i$.

Lemma 2. Let $\left\{r_{n}\right\}_{n=0}^{\infty}$ be an enumeration of $J$, the set of rationals in $I$. Then there exists a 1-1 function $f$ from $c \times \omega_{0}$ into I such that $\lim _{n \rightarrow \infty} f(\alpha, n)=0$ for each $\alpha$ and, defining $F(\alpha, n)=r_{n}+f(\alpha, n), F$ is a 1-1 function from $c \times \omega_{0}$ onto $I-J$.

Proof. Let $S$ be a well ordering of $I$ by $c$ (the first uncountable ordinal). Let $\left\{z_{\alpha}\right\}_{\alpha<c}$ be a well ordering of $c \times \omega_{0}$. Let $k(\alpha)$ be the second coordinate of $z_{\alpha}$. Pick $y \in I$ so that $r_{k(0)}+y$ is the $S$-first irrational number such that $y<2^{-k(0)}$. Put $f\left(z_{0}\right)=y$.

Now suppose we have defined $f$ at each $z_{\xi}$ with $\xi<\beta$ such that $f\left(z_{\xi}\right)<$ $2^{-k(\xi)}$ and $f$ restricted to $\left\{z_{\xi}: \xi<\beta\right\}$ is $1-1$ into $I-J$. Choose $y$ such that $r_{k(\beta)}+y$ is the $S$-first irrational number not in $\left\{f\left(z_{\xi}\right): \xi<\beta\right\}$ and such that $y<2^{-k(\beta)}$. Put $f\left(z_{\beta}\right)=y$. The inductive hypothesis is clearly satisfied. The conclusion of the lemma now follows easily.

Theorem 2. There exists a 1-1 function $h$ from $I$ into $I$ such that for any distinct points $x$ and $y$ in $I$

$$
\left\{\left|h^{n}(x)-h^{n}(y)\right|\right\}_{n=1}^{\infty} \quad \text { is dense in } I .
$$


Proof. Apply Lemmas 1 and 2 to obtain $g$ and $f$. Define $h$ as follows

$$
h(x)= \begin{cases}g(x), & \text { if } x \in J, \\ g\left(r_{n}\right)+f\left(\alpha, r^{-1}\left(g\left(r_{n}\right)\right),\right. & \text { if } x=r_{n}+f(\alpha, n) .\end{cases}
$$

By Lemma $2 h$ is well defined and 1-1 on $I$. If $x=r_{n}+f(\alpha, n)$, then for any $i$ we have

$$
h^{i}(x)=g^{i}\left(r_{n}\right)+f\left(\alpha, r^{-1}\left(g^{i}\left(r_{n}\right)\right)\right) .
$$

By (6) of Lemma $1 \lim _{i \rightarrow \infty} r^{-i}\left(g\left(r_{n}\right)\right)=\infty$ for each $n$. Hence, by Lemma $2 \lim _{i \rightarrow \infty} f\left(\alpha, r^{-1}\left(g^{i}\left(r_{n}\right)\right)\right)=0$ for each $n$.

Now let $\lambda$ be any point in $I$ and let $x$ and $y$ be distinct points in $I$. If $x$ and $y$ are both rational then we have the desired conclusion from Lemma 1. In case $x$ and $y$ are both irrational then $x=r_{n}+f(\alpha, n)$ and $y=$ $r_{m}+f(\beta, m)$ for some $n, m, \alpha$, and $\beta$. We know by Lemma 1 that there exists a subsequence $\left\{i_{k}\right\}_{k=0}^{\infty}$ such that $\left|g^{i_{k}}\left(r_{n}\right)-g^{i_{k}}\left(r_{m}\right)\right| \rightarrow \lambda$. Then $\mid h^{i_{k}}(x)-$ $h^{i_{k}}(y)|=| g^{i_{k}}\left(r_{n}\right)-g^{i_{k}}\left(r_{m}\right)+f\left(\alpha, r^{-1}\left(g^{i_{k}}\left(r_{n}\right)\right)\right)-f\left(\beta, r^{-1}\left(g^{i_{k}}\left(r_{m}\right)\right)\right) \mid$ obviously approaches $\lambda$.

In case either $x$ or $y$ is rational the obvious simplification of the above argument works. This completes the proof.

Note that neither the function $g$ of Lemma 1 nor the function $h$ of Theorem 2 is a surjection. (Footnote: The referee has supplied a different proof of Lemma 1 rendering $g$ a 1-1 continuous function from $J$ onto $J$. Hence, the function $h$ of Theorem 2 can be made a surjection.) It would be interesting to know if the function $h$ of Theorem 2 could be made Baire $\alpha$ for some $\alpha$. Our methods of construction do not seem at all amenable to modification to make $h$ Baire $\alpha$ or even Lebesgue measurable.

Also note that the proofs of Lemmas 1 and 2 as well as Theorem 2 do not depend on the linearity of $I$. Hence, Theorem 2 could be recast in an obvious way to apply to more general spaces such as the closed unit disc or $I \times I$.

\section{BIBLIOGRAPHY}

1. A. M. Bruckner, J. G. Ceder, and T. L. Pearson, On w-limit sets for various classes of functions, Real Analysis Exchange 15 (1990), 592-604.

2. A. M. Bruckner and T. Hu, On scrambled sets for chaotic functions, Trans. Amer. Math. Soc. 301 (1987), 289-297.

3. K. Kuratowski, Topology, Vol. 1, Academic Press, New York, 1966.

4. L. Y. Li and J. Yorke, Period three implies chaos, Amer. Math. Monthly 82 (1975), 985-992.

Department of Mathematics, University of California, Santa Barbara, California 